\title{
Gestão em saúde como competência médica: um relato de experiência
}

\author{
Health management as medical competence: an experience report
}

Gestión de la salud como competencia médica: un informe de experiencia

Hilda Juliana Matieli ${ }^{1}$, Jéssica Faria de Castro Assunção Martins ${ }^{1 *}$, Vitória Lopes Cançado Assis ${ }^{1}$, Domitila Natividade Figueiredo Lopes ${ }^{1}$, Maíra Ferro de Sousa Touso¹.

\section{RESUMO}

Objetivo: Compreender o processo formativo do estudante de medicina, a fim de capacitar o futuro médico para a atuação em gestão na Atenção Primária à Saúde (APS). Relato de experiência: Trata-se de relato de experiência reflexivo, conduzido por 3 acadêmicas de medicina do quinto ano de uma universidade privada no interior de São Paulo. Confeccionado a partir das vivências no processo formativo e suas inserções na APS deste o primeiro ano da graduação, compreendendo a atuação das mesmas na implantação de um Conselho Gestor (CG) de uma UBS (Unidade Básica de Saúde). A fim de refletirem sobre os êxitos e insucessos desta experiência rumo à formação de um médico generalista, ético, empático e com habilidades em gestão de saúde, elegeram três categorias temáticas para a discussão. Dentre as dificuldades elencaram a baixa literacia em saúde da comunidade assistida, a marginalidade do tema nos discursos acadêmicos e, enquanto possibilidade a prática na reimplementação de um CG e entendimento decorrente da importância do controle social. Considerações finais: A reflexão demonstrou a necessidade do fortalecimento da temática nos currículos das graduações médicas para o sucesso do imprescindível o papel do médico como líder nas atividades políticas e de planejamento em saúde.

Palavras-chave: Educação médica, Conselho de planejamento de saúde, Atenção primária à saúde.

\begin{abstract}
Objective: To understand the importance of the medical student's role as a future Primary Care manager in a city of São Paulo, Brazil. Experience report: This is a reflective experience report, conducted by 3 medical students at the University of Franca - São Paulo, based on their performance at the implementation of the Management Council (CM) of Basic Health Unit. The students identified the curriculum deficiency at medical graduation of health managers. As result, it is important to encourage the contact with health management, during the medical graduation, in order to ability academics to be able to solve problems and improve the community social context. Final considerations: The reflection demonstrated the need for restructuring the medical curriculum at universities, since the physician shows an important role as a leader in political and health planning activities that are not being explored during graduation. CM is a great advance in developing a better medical graduation, although, adherence is usually low due to the precarious health literacy of the assisted community.
\end{abstract}

Keywords: Medical education, Management council, Primary health care.

\section{RESUMEN}

Objetivo: Comprender la importancia del papel del estudiante de medicina como futuro gerente en atención primaria en una ciudad de São Paulo, Brasil. Informe de experiencia: Es un informe de experiencia reflexivo, realizado por 3 estudiantes de medicina de la Universidad de Franca, São Paulo, basado em su

${ }^{1}$ Universidade de Franca (UNIFRAN), Franca - SP. *E-mail: jessicaassuncao1@hotmail.com 
desempeño en la implementación del Consejo de Administración (CG) de la Unidad Básica de Salud (UBS), habiendo sido identificado la deficiencia en el plan de estudios sobre la graduación de gerentes médicos durante el curso. Como resultado, es importante el contacto académico con la gestión de la salud para la posibilidad de resolución de problemas y mejora de lo social en la comunidad. Consideraciones finales: La reflexión demostró la necesidad de reestructuración del plan de estudios de las universidades médica, ya que el papel del médico como líder en las actividades políticas y planificación de la salud es esencial, siendo poco explorado durante la graduación. Aunque las CG son grandes avances en la construcción de un sistema de mejor cualidad, la adherencia generalmente es baja, visto la precaria graduación en salud de la comunidad asistida de los alumnos.

Palabras clave: Educación médica, Consejo de administración, Atención primaria de salud.

\section{INTRODUÇÃO}

Aprovada pela Assembleia Nacional Constituinte, em 22 de outubro de 1988, a Constituição Cidadã, marcou o fim do período do governo autoritário, nacionalista e repressor dos direitos humanos e sociais, chamado de Ditadura Militar (1964-1985), e o início do processo de redemocratização, chamado de Nova República, fundamentada na soberania, na cidadania e na dignidade do indivíduo (LARA R e SILVA MA, 2015; BRASIL, 2019).

A partir disso, era determinada a igualdade de todos perante a lei, garantindo a inviolabilidade do direito a vida, a liberdade, a segurança e a propriedade. Com isso, foi visto como necessária a reestruturação não apenas do que refletia nos Poderes da União com o Executivo, o Legislativo e o Judiciário, mas também no âmbito da saúde (STF, 2018). Logo, em 1990, foi decretada e sancionada a Lei no 8.080 que visava as seguintes funções do Estado: a de promoção, a de proteção, a de recuperação da saúde, a de organização e dos funcionamentos dos serviços a ela correspondentes, a partir da criação do Sistema Único de Saúde (SUS) (CASA CÍVIL, 1990).

Sendo uma das maiores conquistas consagradas, esse sistema transformou a medicina como "o Estado de não doença" em um modelo direcionado à qualidade de vida do indivíduo englobando todos os conceitos de saúde, bem-estar físico, mental e social (BRASIL, 2003).

No entanto, um dos desafios é o controle social que "é a produção de necessidades da vida por seus próprios protagonistas. É acima de tudo partilhar poder. Construção de um processo político pedagógico de conquista da cidadania e fortalecimento da sociedade civil" (BRASIL, 2015), resultando na atuação da comunidade no processo de formulação das políticas públicas no âmbito da saúde.

Para isso, são formados conselhos com atribuição e competência das três esferas políticas e administrativas. Assim, é visto o limite de atuação dos gestores que trabalham através de um conjunto de princípios coerentes com a diversidade operativa de cada território, identificando as necessidades locais e as resolvendo de forma dinâmica (BRASIL, 2015).

Um mecanismo facilitador nesse processo foi a criação do Conselho Gestor (CG) dentro do ambiente das Unidades Básicas de Saúde (UBS), atuando como um canal de "participação que articula representantes da população e membros do poder público em práticas que dizem respeito à gestão de bens públicos" (GOHN MG, 2012), com o objetivo de implantar políticas para a redução de índices de pobreza, de desigualdade socioeconômica, de gênero e de etnia e das questões ambientais que interferem na saúde. Portanto, é realizada uma análise da realidade local com identificação dos problemas de base e dos potenciais perigos para formular propostas de intervenções e resoluções dos problemas visibilizados (BRASIL, 2015).

Concomitantemente, a gestão social é um facilitador da prática cidadã e um espaço compartilhado entre a sociedade civil e o governo. Logo, a formação do CG leva em consideração três segmentos importantes nas ações e políticas da área da saúde que são o Usuário, os membros da população que usufruem da UBS, o Trabalhador, aqueles indivíduos prestadores de serviços da unidade independente do vínculo 
empregatício; e o Gestor, os trabalhadores em cargos de gerência das unidades básicas, sendo com composição de $50 \%$, 25\% e 25\%, respectivamente escolhidos por eleição, tendo como foco de interesse os problemas locais e não pessoais (GOMES EGM, 2003).

Para isso, é imprescindível a atuação do médico como líder no processo de formulação e planejamento de políticas dentro da saúde a fim de tornar mais eficiente e integrada a APS. Com isso, torna-se necessário a educação em gestão de saúde nos primórdios da formação médica explorado na graduação (FARIAS LABG, 2018).

Segundo as Diretrizes Curriculares Nacionais (DCNs), o médico como generalista, humanista, crítico e reflexivo deve ser capacitado para atuar nos diversos níveis de atenção, focando na promoção, prevenção, recuperação, reabilitação da saúde e na intervenção da gestão para a melhora das políticas públicas, pautados na integralidade e interdisciplinaridade (FARIAS LABG, 2018). No entanto, vê-se esse engajamento na formação acadêmica de forma deficitária e insuficiente para a formação do estudante enquanto gestor e facilitador do controle social, sendo necessário aprimorar a inserção deste tema na formação.

Diante disso, este estudo buscou relatar a experiência de quatro acadêmicas de medicina, inscritas no sexto ano de uma universidade do interior de São Paulo, na realização de um processo reflexivo estabelecido à fim de pensar no processo formativo em suas competências e insuficiências na capacitação do estudante para sua futura atuação na gestão em saúde.

\section{RELATO DE EXPERIÊNCIA}

O delineamento do estudo é descritivo, do tipo relato de experiência, retrospectivo, utilizando para tanto, o resgate dos conteúdos dos portfólios reflexivos construídos do primeiro ao quarto ano, assim como discussões grupais, no estágio de saúde da família, no quinto ano.

$\mathrm{Na}$ faculdade de origem das estudantes e docentes deste estudo, semanalmente, acontecem as práticas de um estágio que promove a integração acadêmica com os cenários da APS, do primeiro ao quarto ano. A duração semanal das práticas é de 5 horas/aula, os grupos de seis acadêmicos são acompanhados por uma docente e realizam práticas de complexidade progressiva, tendo como eixo central a PS da população adscrita.

No quinto ano, a temática APS voltou ao campo de práticas por meio do estágio do internato em Saúde da Família, em uma UBS mista que incorpora a Estratégia de Saúde da Família, da região norte da cidade, durante 6 semanas do ano letivo. Neste, os estudantes acompanhados de um docente supervisor, aplicam os preceitos da medicina da família em atendimentos clínicos, domiciliares, estratégias de PS nos equipamentos sociais do território e são realizadas discussões grupais diárias.

No início da trajetória destas acadêmicas, no primeiro e no segundo da graduação, acompanhadas de uma docente apta a compreender o indivíduo e os determinantes sociais, foi reinstalado o CG, dentro da UBS. Para isso, notou-se a necessidade de atividades de educação em saúde, então realizadas sobre o conselho. Porém, o mesmo deixou de operar após dois anos. Estabeleceu-se, portanto, este relato visando refletir sobre possíveis falhas na sua conservação e atuação e, consequentemente, compreensão dos subsídios imprescindíveis na formação e a capacitação do estudante, futuro médico, no papel de gestor.

A participação na organização, proporcionou às estudantes a oportunidade de articular a teoria aprendida com a prática vivenciada, refletindo sobre as possibilidades de autonomia oriundas da divulgação de conhecimento, através de ações de educação em saúde, objetivando a participação social ativa e promoção de melhorias na atenção à saúde.

Evidenciou-se que a divulgação de informações contribuiu na adesão, na busca de informações sobre o $C G$ e na efetividade da eleição, sendo que todas as vagas foram preenchidas e as reuniões continuaram acontecendo mensalmente naquele ano e no subsequente, entretanto, por carência de incentivos e capacitação contínua dos membros, o mesmo, mais uma vez deixou de atuar. 
Na experiência vivenciada e exitosa, a PS e prevenção de doenças, tendo como cenário a APS, são práticas necessárias e relevantes. No entanto, pôde-se notar que a população adscrita tinha pouco acesso às atividades realizadas na UBS que iam além do modelo curativo e clínico. Contudo, possibilitou a ponderação acerca dos fatores que podem influenciar na não funcionalidade e adesão da comunidade no conselho por meio do controle social e da gestão de saúde, além de fomentar a compreensão das ações da academia na comunidade para o aperfeiçoamento da APS e valorização das políticas que fomentam a PS e cuidado integral da pessoa.

Além disso, a extinção do CG, apontou uma irrisória participação da comunidade nas reuniões, gerando pouca atuação nas campanhas, dificultando o entendimento do usuário em relação à APS e seu papel necessário enquanto protagonista na gestão e PS; assim como uma necessária mudança nas premissas dos profissionais de saúde para a adequada valorização do SUS e implementação efetiva do controle social.

Depreende-se desta observação a percepção da baixa literacia em saúde desses. Segundo a OMS, a literacia é definida como um rol de habilidades cognitivas e sociais que determinam a motivação e habilidade dos indivíduos para obter acesso, entender e usar a informação de forma a promover e manter a boa saúde. Compreendendo os determinantes sociais da saúde, o indivíduo pode otimizar tanto a sua saúde quanto a de seus familiares e da comunidade, caminhando para a PS.

Assim sendo, mostra-se imprescindível que as faculdades médicas cumpram as DCNS a fim de proporcionar ao estudante vivências significativas na APS, assim como capacitem seus docentes para a compreensão da saúde ampliada e do necessário protagonismo médico para uma gestão eficiente do SUS, respeitando seus princípios e políticas.

\section{DISCUSSÃO}

A análise das experiências ocorreu inspirada pelo Método de Interpretação de Sentidos, o qual propõe uma interpretação para além dos conteúdos de textos, aprofundando-se nos contextos. Procura-se, assim, a articulação buscando uma compreensão (hermenêutica) e uma crítica (dialética) a partir da formação acadêmica das estudantes (GOMES R, 2007).

\section{Categoria de análise: Literacia em Saúde}

Dentre as habilidades fundamentais para a literacia, devem constar: aptidão em utilizar tecnologia, capacidade cognitiva, apresentar motivação, trabalho em rede e habilidades sociais. É possível promovê-la articulando nos diversos setores da sociedade a educação para a saúde e a cultura da PS., No entanto, a literatura aponta que apesar das tentativas para melhorar e despertar o interesse da população por conhecimentos sobre a saúde e para melhorar a transmissão de informações, em muitos momentos os instrumentos não são ideais, podendo trazer informações alteradas, não reduzindo as disparidades entre os resultados dos cuidados de saúde (QUEMELO PRV, et al., 2017).

Visto que a mesma está relacionada a capacidade de obter, processar e compreender as informações necessárias para as decisões quanto à saúde, os mais propensos a serem portadores de baixa literacia têm como perfis: menor nível socioeconômico e educacional, adolescentes e idosos. Esta população está mais predisposta a baixa adesão ao tratamento; pequena promoção de saúde; redução do uso de serviços preventivos; gestão ineficaz de condições crônicas; maior propensão à erros médicos, tempo de internação e maiores taxas de mortalidade (TOMÁS C, et al.,2015; JUNIOR JCB e RIGOLIN CCD, 2014).

A partir disso, não se deve focar no estilo de vida e no modo de utilização dos serviços de saúde, mas favorecer as ações que visam o empoderamento (JUNIOR JCB e RIGOLIN CCD, 2014). A educação permanente em saúde é uma estratégia de treinamento profissional, desenvolvendo a interdisciplinaridade no trabalho, reduzindo as fragmentações dos serviços e dos saberes que pautam as ações, através do ensino-aprendizagem problematizadora, incorporando $o$ debate e a aquisição de conhecimento e desenvolvimento de atitudes promotoras de literacia (FARINELLI MR, et al., 2017). 
Logo, depreende-se desta reflexão a necessidade premente de mudança cultural da população, assim como dos trabalhadores de saúde para que o protagonismo do usuário seja central e a cultura da saúde suplante a da doença. A escola médica enquanto uma instância promotora da PS em seus cenários de práticas seria uma aliada neste processo e teria um compromisso social.

\section{Categoria de análise: Marginalidade do tema dos discursos na formação}

O ensino médico, foi amplamente criticado quanto à sua capacidade de desenvolver no estudante o potencial intelectual e de julgamento, da abordagem de análise e de raciocínio crítico, resultando na necessidade de reestruturação em curso do ensino

Surgindo, então, a noção de Quadrilátero de Formação com a proposta de exaltar as características locais, valorizando as capacidades instaladas, desenvolvendo as potencialidades existentes em cada realidade e estabelecendo a aprendizagem significativa e crítica. Tal proposta pretende suplantar a concepção hegemônica tradicional (biologicista, mecanicista, centrada no professor e na transmissão) para uma concepção construtivista (problematizadora das práticas e dos saberes, centrada no estudante), incentivando a construção de conhecimento e tendo como cenários importante a APS (FERREIRA RC, et al., 2010).

De acordo com as diretrizes curriculares, da formação espera-se o desenvolvimento de conhecimentos, habilidade e atitudes do acadêmico com o intuito de fomentar o protagonismo médico, com aptidão e competência geral de gerenciar e administrar os recursos físicos, materiais, informacionais, além de aptidões enquanto gestores ou empregadores das equipes de saúde (FARIAS LABG, 2018).

Apesar das mudanças em curso, na rotina acadêmica, notou-se que os estudantes ao integrarem teoria e pratica permanecem fragmentando o aprendizado, levando à manutenção da ideia da prática em especialidades. Também os docentes, muitos especialistas, corroboram em seus discursos esta fragmentação e o ideal da integralidade e PS se distancia da formação.

Diante desse cenário, destaca-se a necessidade de que as mudanças currículos dos cursos da saúde com conceitos e práticas relacionados à organização, à promoção da saúde (PS) e à prevenção de doenças com a utilização de metodologias de ensino-aprendizagem ultrapassem os espaços acadêmicos (FERREIRA RC, 2010). Faz-se necessário, portanto, a formação de docentes da faculdade médica aptos a formarem os estudantes nessas premissas e que compreendam a saúde enquanto um conceito ampliado e a importância dos mecanismos de gestão, desde os anos iniciais aos finais da graduação.

A função do docente sustenta as atividades educativas que se iniciam quando o estudante se familiariza com o novo processo que passa a vivenciar. Esta prática educativa o estimula a expor suas dúvidas, sentimentos, conhecimentos, valores e ideias, sem medo de ser julgado como acontece no ensino tradicional. O trabalho pedagógico deve fluir estimulando a trajetória e possibilitando o desenvolvimento de capacidades para o trabalho em saúde, o reconhecimento das próprias dificuldades e a elaboração de propostas para superá-las. Além disso, o papel do docente é essencial para o cumprimento das DCNS pelas faculdades de medicina (FERREIRA RC, 2010; FARIAS LABG, 2018).

A inserção destes docentes e estudantes, integrados com os profissionais dos serviços de saúde é de grande valia, pois assim desempenhariam o papel de supervisores dos estudantes, auxiliando para a organização das práticas e para o desenvolvimento conjunto das atividades. Por fim, é de suma importância o envolvimento do acadêmico em medicina na busca pelo ensino e inserção da gestão no curso médico (FERREIRA RC, 2010; FARIAS LABG, 2018). A interdisciplinaridade, vivida e aprendida, promoveria aos estudantes que se formarão, os fundamentos da gestão em saúde.

\section{CONSIDERAÇÕES FINAIS}

A partir da precária ênfase da temática gestão em saúde nas faculdades médicas, as DCNs enfatizam a necessidade da reestruturação da formação à fim de formar um profissional generalista, humanista, que compreenda a integralidade do cuidado; apto a exercer a liderança e gestão nos serviços de saúde. A 
exemplo de um instrumento facilitador do tripé do controle social, literacia e gestão em saúde, tem-se a aplicabilidade do CG atuante para a melhoria das condições de saúde de uma comunidade. A experiência relatada favoreceu a consolidação do conhecimento teórico-prático acerca do necessário protagonismo médico para uma gestão eficiente do SUS, respeitando seus princípios e políticas. Assim como, possibilitou a compreensão de que a formação profissional e interação dos serviços com as comunidades são essenciais para a efetividade das políticas públicas de saúde.

\section{REFERÊNCIAS}

1. LARA R, SILVA MA. A ditadura civil-militar de 1964: os impactos de longa duração nos direitos trabalhistas e sociais no Brasil. Serviço Social \& Sociedade, 2015; 122:275-293.

2. BRASIL. Constituição da República Federativa do Brasil. Brasília: Supremo Tribunal Federal, Secretaria de Documentação, 2019.

3. CASA CIVIL. Lei $n^{\circ} 8.080$, de 19 de setembro de 1990, dispõe sobre as condições para a promoção, proteção e recuperação da saúde, a organização e o funcionamento dos serviços correspondentes e dá outras providências. Presidência da República, 1990.

4. BRASIL. Conselho Nacional de Secretários de Saúde. Legislação do SUS / Conselho Nacional de Secretários de Saúde. - Brasília: CONASS, 2003. 604 p.

5. ALBERTON V, et al. Artigo - Conselho gestor: uma alternativa para o desenvolvimento regional. FACCREI; 2017.

6. BRASIL. Conselho Nacional de Secretários de Saúde. A Gestão do SUS / Conselho Nacional de Secretários de Saúde. Brasília: CONASS, 2015. 133 p.

7. GONH MG. Novas teorias dos movimentos sociais. 4. ed. São Paulo: Loyola, 2012.

8. GOMES EGM. Conselhos gestores de políticas públicas: democracia, controle social e instituições. Dissertação (Mestrado em Administração Pública e Governo) - Escola de Administração de São Paulo. Fundação Getúlio Vargas, São Paulo, 2003; 112 p.

9. GOMES R. Análise e interpretação de dados de pesquisa qualitativa. In: MINAYO, M.C.S. (ORG). Pesquisa social: teoria, método e criatividade. Petrópolis: Vozes, 2007. p. 79-108.

10. QUEMELO PRV, et al. Literácia em saúde: tradução e validação de instrumento para pesquisa em promoção da saúde no Brasil. Cadernos de Saúde Pública, 2017; 33(2).

11. FERREIRA RC, et al. Formação profissional no SUS: o papel da Atenção Básica em Saúde na perspectiva docente. Revista Brasileira de Educação Médica, 2010; 34(2):207-215.

12. FARIAS LABG. Gestão em saúde e escolas médicas: estudantes de medicina como futuros gestores. Revista de Administração em Saúde, 2018; 18(70).

13. TOMÁS C, et al. Impacto da literacia em saúde nos comportamentos promotores de saúde em gestão de stresse em adolescentes. Rev Port de Enf de Saúde Mental 2015; 2: 101-106.

14. JUNIOR JCB, RIGOLIN CCD. Inter-relações entre literacia em saúde e a comunicação pública da ciência. II semana de pós-graduação em ciência política - Universidade Federal de São Carlos, São Carlos, 2014.

15. FARINELLI MR, et al. Permanent education and health literacy: contributions for the training professional. REFACS 2017; 2(5): 305-310. 